\title{
Thoracic ultrasonography: a narrative review
}

\author{
P. H. Mayo ${ }^{1 *} \mathbb{D}$, R. Copetti ${ }^{2}$, D. Feller-Kopman ${ }^{3}$, G. Mathis ${ }^{4}$, E. Maury ${ }^{5,6,7}$, S. Mongodi ${ }^{8}$, F. Mojoli ${ }^{8}$, G. Volpicelli ${ }^{9}$ \\ and M. Zanobetti ${ }^{10}$
}

(c) 2019 Springer-Verlag GmbH Germany, part of Springer Nature

\begin{abstract}
This narrative review focuses on thoracic ultrasonography (lung and pleural) with the aim of outlining its utility for the critical care clinician. The article summarizes the applications of thoracic ultrasonography for the evaluation and management of pneumothorax, pleural effusion, acute dyspnea, pulmonary edema, pulmonary embolism, pneumonia, interstitial processes, and the patient on mechanical ventilatory support. Mastery of lung and pleural ultrasonography allows the intensivist to rapidly diagnose and guide the management of a wide variety of disease processes that are common features of critical illness. Its ease of use, rapidity, repeatability, and reliability make thoracic ultrasonography the "go to" modality for imaging the lung and pleura in an efficient, cost effective, and safe manner, such that it can largely replace chest imaging in critical care practice. It is best used in conjunction with other components of critical care ultrasonography to yield a comprehensive evaluation of the critically ill patient at point of care.
\end{abstract}

Keywords: Ultrasonography, Lung ultrasonography, Pleural ultrasonography, Critical care ultrasonography

\section{Introduction}

Thoracic ultrasonography is a key part of critical care ultrasonography (CCUS), as it allows the intensivist to examine the lung and pleural space. It can reduce the use of standard chest radiography (CXR) and computerized tomography (CT) in the intensive care unit (ICU) $[1,2]$. Its ease of use, rapidity, repeatability, and reliability make thoracic ultrasonography the "go to" modality for imaging the lung and pleura in an efficient, cost effective, and safe manner. The practical constraints and risks of transporting the critically ill patient to the CT scanner and the inevitable associated radiation exposure make thoracic ultrasonography, whenever possible, a particularly attractive alternative the chest $\mathrm{CT}$. This article will review technical aspects of thoracic ultrasonography, followed by a discussion of some of its indications. This article will assume that the reader has familiarity with the standard

\footnotetext{
*Correspondence: mayosono@gmail.com

${ }^{1}$ Division of Pulmonary, Critical Care and Sleep Medicine, Northwell Health, Zucker School of Medicine at Hofstra/Northwell, New Hyde Park, Hempstead, NY 11549, USA

Full author information is available at the end of the article
}

semiology of thoracic ultrasonography that is well summarized in widely available textbooks and consensus statements [3-6]. The reader is invited to review the electronic supplementary video material that demonstrates some typical findings of thoracic ultrasonography.

\section{Technical aspects of thoracic ultrasonography}

Lung and pleural ultrasonography can be performed with most recent generation ultrasonography machines. The smaller machines that are well suited for critical care work yield adequate images. Image quality is determined by the individual machine characteristics, presets, and post-image processing. Lung ultrasonography often relies on analysis of artifacts, such as A- and B-lines, which can be optimized by altering machine settings; the experienced operator adapts machine settings for optimal visualization of these artifacts [7]. Some manufacturers have included presets specifically for lung ultrasonography. These are not necessarily the best settings, so the intensivist needs to adjust the machine settings for optimal image quality. This may require adjusting the gain or altering the post-processing settings of the machine.

Thoracic ultrasonography images may be acquired with a curvilinear probe designed for abdominal

\section{县


ultrasonography. Alternatively, a phased array probe designed for cardiac imaging may be readily configured for thoracic ultrasonography. This reduces the cost of equipment, as one probe serves two purposes. The phased array probe has the added advantage of a small footprint to better scan between rib interspaces. A high frequency linear probe designed for vascular access guidance is useful for detailed imaging of the pleural line.

There is no best way to perform image acquisition for thoracic ultrasonography. One elegant method utilizes three pre-determined examination points on the left hemithorax and three identical pre-determined examination points on the right hemithorax. This yields enough information to categorize the cause of acute respiratory failure in a high percentage of case. This approach is codified in the BLUE protocol (Fig. 1; Table 1) [8-10]. Other scanning protocols have been described in a definitive guideline document [4]. An alternative method is for the intensivist to examine multiple adjacent rib interspaces while moving the probe in linear fashion across the thorax in a series of longitudinal scan lines. This yields a comprehensive view of the thorax. If an abnormality is detected, the operator focuses on the area of interest in more detail. There is no mandatory method of performing the thoracic ultrasonography examination. This reflects on the flexibility of the technique which is controlled by the clinician and applied according to the clinical requirements of the case.

Thoracic ultrasonography has several limitations. It requires the intensivist to be competent in image acquisition, image interpretation, and the cognitive base. While the cognitive base is well described, the required training sequence for image acquisition has not been well validated. General guidelines have published regarding the training methods that are recommended for general critical care ultrasonography that may be adapted to thoracic

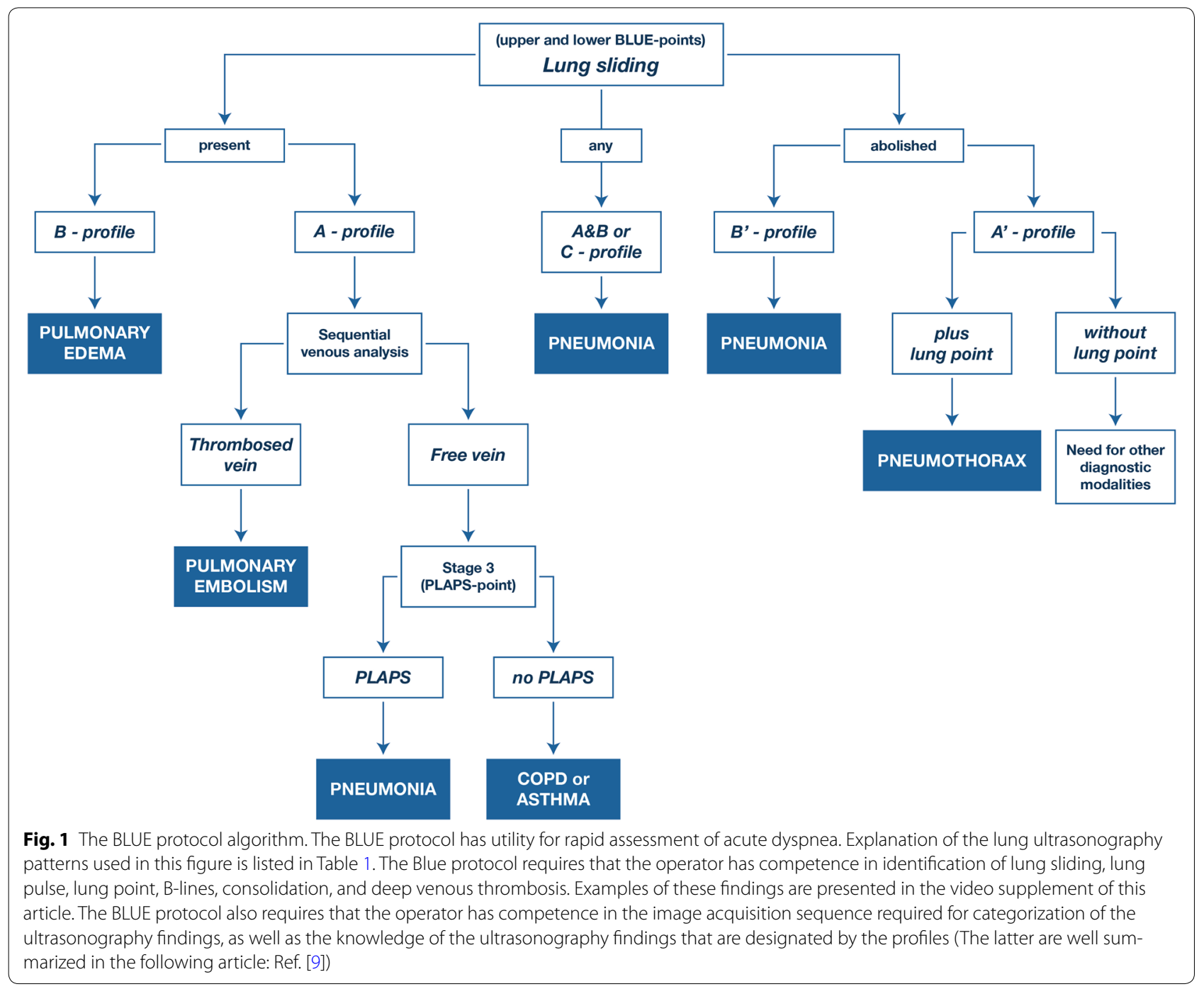


Table 1 Lung ultrasonography findings of ACPE and ARDS

\begin{tabular}{|c|c|c|}
\hline & ACPE & ARDS \\
\hline Clinical setting & Acute & Acute \\
\hline B-lines & Always present & Always present \\
\hline Distribution of B-lines & Bilateral and symmetric distribution & $\begin{array}{l}\text { Non-homogeneous distribu- } \\
\text { tion, presence of spared } \\
\text { areas }\end{array}$ \\
\hline Pleural line abnormalities & Absent & Present, typical \\
\hline Reduction or absence of lung sliding & Absent & Present \\
\hline Lung pulse & Absent & Present \\
\hline Consolidations & Absent & Frequent in the posterior areas \\
\hline
\end{tabular}

$A C P E$ acute cardiogenic pulmonary edema, $A R D S$ adult respiratory distress syndrome

ultrasonography [11]. The studies referenced the present article have been performed by groups with a high-level capability in lung ultrasonography. The reader can only expect to achieve similar results, if they have a similar level of competence. Another limitation is that aerated lung blocks transmission of ultrasound, so any process within the lung that is surrounded by aerated lung will not be visualized by thoracic ultrasonography. Similarly, a pneumothorax blocks visualization of underlying lung. A specific failure point of thoracic ultrasonography performed in the critically ill patient occurs in the supine patient, as it may be difficult to fully image the posterior thorax. In this case, the probe is placed as posterior as possible with angulation of the tomographic plane toward central body mass. Occasionally, it may be necessary to place the patient in a lateral decubitus position for full imaging of the posterior thorax. Patient-specific factors such as obesity, edema, chest drains, or dressings may degrade the image quality of thoracic ultrasonography.

\section{Ultrasonography for the evaluation of pneumothorax}

Mastering this application is a key skill for the intensivist, as it is superior to chest radiography for detection of pneumothorax when compared to antero-posterior CXR that are commonly used for evaluation of the critically ill and trauma patient population in whom lung ultrasonography for evaluation of pneumothorax is well validated. For pneumothorax, ultrasonography had a pooled sensitivity of $78.6 \%$ (95\% CI 68.1-98.1) and a specificity of $98.4 \%$ (95\% CI 97.3-99.5). Chest radiography had a pooled sensitivity of $39.8 \%$ (95\% CI 29.4-50.3) and a specificity of $99.3 \%$ (95\% CI 98.4-100) [12]. Evaluation of pneumothorax requires knowledge of several signs of lung ultrasonography including lung sliding, lung pulse, lung point, and B-lines (Fig. 2). M-mode may be used to supplement 2-D imaging [13]. The presence of lung sliding rules out pneumothorax at the site of probe application to the chest wall [14]. However, the absence of lung sliding does not necessarily indicate a pneumothorax. For example, pleurodesis, severe parenchymal lung disease, ARDS with low tidal volume ventilation, giant bullae, or bronchial occlusion will all ablate lung sliding.

While the absence of lung sliding suggests the possibility of pneumothorax, the presence of a lung point is diagnostic of a pneumothorax. A lung point is found at the intersection of the partially deflated lung and the airfilled pneumothorax space, where the partially collapsed lung moves in and out the tomographic plane of the ultrasonography probe in respirophasic manner. The lung point is $100 \%$ specific but only $60 \%$ sensitive for pneumothorax [15]. A lung point will be absent in complete pneumothorax where the lung is completely retracted to the hilum. The location of the lung point may be used to provide a semi-quantitative estimate of the size of a pneumothorax [16]. In this case, the examiner may identify a lung point at several adjacent rib interspaces to find the lateral extent of the pneumothorax while the patient is in supine position. With a small pneumothorax, the lung point will be located over the anterior chest. With a larger pneumothorax, the lung point will be located more laterally. This allows the examiner to map out the lateral extent of the pneumothorax space.

The presence of a lung pulse signifies that the visceral and parietal pleura are in apposition at the site of probe application to the chest wall, so its presence rules out pneumothorax at the site of probe application to the chest wall. Lung pulse is present without lung sliding in the case of an atelectatic lung, as might occur with a right mainstem intubation. In the case of right mainstem intubation, lung sliding is present on the right side; while on the left, there is lung pulse but no lung sliding [17]. The presence of B-lines or short vertical artifacts at the site of probe application to the chest wall rules out pneumothorax [18]. The observation of 


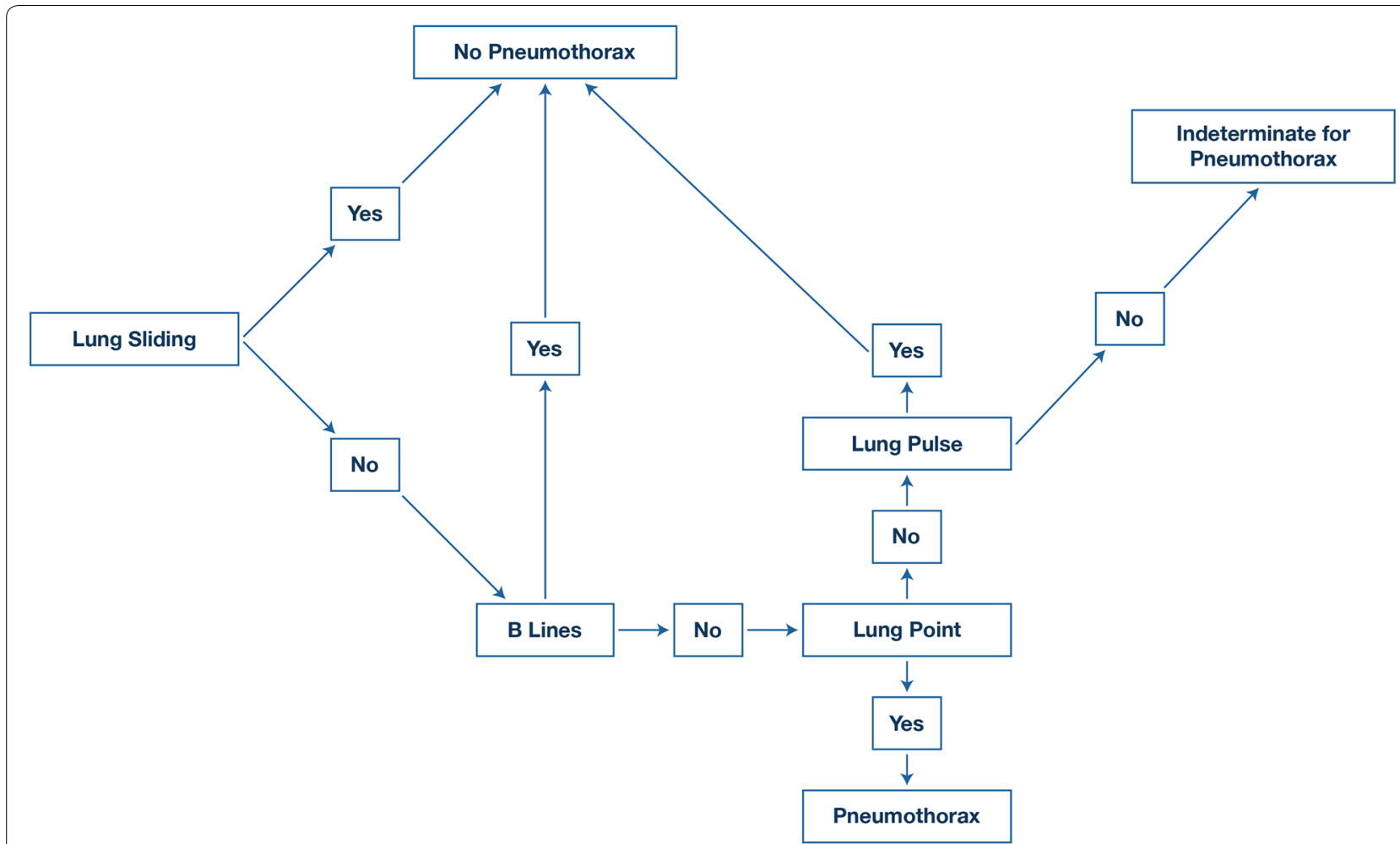

Fig. 2 Evaluation for pneumothorax using lung ultrasonography. This algorithm requires that the operator has competence in identification of lung sliding, lung pulse, lung point and B-lines. Examples of these findings are presented in the video supplement of this article

consolidation or pleural effusion rules of pneumothorax at the site of probe application to the chest wall as the presence of even a small amount of pleural air will obscure the underlying lung.

Only a short period of training is required to accurately examine the patient for pneumothorax with one study showing that a dedicated training class of 2 hours duration was sufficient to train emergency medicine (EM) physicians for identification of pneumothorax [19]. The intensivist can rapidly assess for pneumothorax by scanning the rib interspaces of the anterior and lateral chest: as a pneumothorax exclusively located in the posterior part of chest is uncommon. Vigorous intercostal muscle contraction can cause traction movement of the parietal pleural surface that may be mistaken for lung sliding.

Ultrasonography is useful for management of the pleural drainage device that has been inserted to treat a pneumothorax. Initially, lung ultrasonography is used to identify the pneumothorax, to target the site for device insertion, and to document re-expansion of the affected lung. Once there is no further air leak, ultrasonography is used to document that the lung remains inflated by ascertaining that lung sliding is present over the anterior chest area. In spontaneous and post-surgery pneumothorax, this strategy of ultrasonography-guided chest tube removal was effective and reduces the need for CXR [20, 21].

Thoracic ultrasonography for evaluation of pleural effusion Pleural effusions are seen in up to $60 \%$ of intensive care unit (ICU) patients and can be associated with increased duration of mechanical ventilation and ICU stay $[22,23]$. Ultrasonography is superior to CXR for determining the presence of pleural fluid, estimating its volume, predicting the characteristics of the fluid, and in guiding pleural intervention [24-29]. Moderate to large pleural effusions are seen in up to one-third of patients at the initiation of ventilator weaning trials and are associated with higher rates of ventilator weaning failure as well as higher 28-day mortality rates [30].

A major application of ultrasonography is for guidance of thoracentesis both for diagnostic and therapeutic purposes. Pleural fluid sampling in critically ill patients has been shown to alter management in over $40 \%$ of patients [22]. The location for a pleural intervention will depend on identifying the largest space between the parietal and visceral pleura well away from the liver or spleen, heart and lung. The operator estimates the size of the effusion [26-28], as well as characteristics of the effusion (i.e., anechoic, hypoechoic, hyperechoic, septated). The 
majority of cases do not require real-time guidance for needle insertion; however, it is crucial to know the distance from the skin to parietal pleura, the distance from the parietal to the visceral pleural and trajectory of needle insertion, as well as the location of the diaphragm, liver/ spleen, heart, lung and diaphragm [31, 32]. The risks of draining pleural effusions with ultrasonography guidance patients on positive pressure ventilation are low, with a reported pneumothorax rate of $1.2-3.4 \%$ [33, 34]. Ultrasonography can also be used to identify intercostal vessels that would contraindicate needle insertion; aberrant intercostal vessel position is most common within $10 \mathrm{~cm}$ of the posterior midline of the thorax [35]. In addition to guidance of thoracentesis, ultrasonography allows the characterization of volume, location, and internal echogenicity of the effusion. Transudates are very likely to be anechoic, whereas an anechoic effusion may be either a transudate or an exudate. The presence of complex septated, complex nonseptated, or homogeneously echogenic patterns indicates a high probability of an exudate [36]. Ultrasonography is superior to chest CT for defining elements within an effusion [37].

\section{Lung ultrasonography versus CXR for evaluation of acute dyspnea}

Many studies have confirmed the accuracy of lung ultrasonography for the identification of diseases that are associated with processes that cause acute respiratory failure. In fact, lung ultrasonography is superior to standard ICU CXR for identification of consolidation, interstitial syndrome, pneumothorax, and pleural effusion in patients on mechanical ventilatory support when chest CT is used as the reference standard [38, 39]. The utility of lung ultrasonography for identification of acute heart failure is well established to be superior to evaluation that uses CXR as the primary imaging modality [40, 41]. The BLUE protocol that combines lung ultrasonography with venous study provided a correct diagnosis in $90.5 \%$ of ICU patients with acute dyspnea [8-10].

In reference to the utility of ultrasonography compared to CXR for the diagnosis of acute dyspnea, an approach using ultrasonography (thoracic, cardiac, deep veins) was superior for evaluation of acute dyspnea in the ED when compared to an approach using CXR as the initial imaging modality [42]. Lung ultrasonography alone was superior in establishing diagnosis and guiding therapeutic intervention for acute dyspnea compared to one that used CXR as the initial imaging modality [43].

In a pilot study of 118 patients, there was good concordance between lung ultrasonography and CXR in establishing a diagnosis for the cause of acute dyspnea [44]. In a prospective follow-up study by the same investigators, (involving 2863 consecutive patients presenting with dyspnea to the ED), the diagnostic accuracy of a standard evaluation was compared to one that incorporated lung ultrasonography, echocardiography, and inferior vena cava evaluation performed at point of care by the ED physician [45]. Standard evaluation included CXR as the initial imaging modality, and/or CT scans, and/or echocardiography performed by a cardiologist, as needed. This was compared to evaluation that utilized ultrasonography as the primary imaging modality. There was no statistically significant difference in the diagnostic accuracy of the two methods, except for acute heart failure diagnosis, where point of care ultrasonography proved to be superior, and the standard approach, which was more accurate for the identification of pulmonary embolism (PE) and COPD/asthma. The main outcome difference between the two methods of evaluation was the time needed to reach diagnosis. Ultrasonography evaluation proved to be significantly time saving with respect to standard approach.

The utility of lung ultrasonography is well studied in the ED with the understanding that the published studies come from groups who are expert level in the field. It has not been well studied in the intensive care unit with the exception of the BLUE protocol (Fig. 2; Table 1) which has strong utility in both the ICU and the ED $[8,46]$. To duplicate the results from the EM literature, intensivists will need to have similar skill level as the EM investigators.

In summary, lung ultrasonography (with addition of echocardiography and venous study) combined with history and physical examination may be an effective alternative to CXR for evaluation of acute dyspnea with chest $\mathrm{CT}$ reserved for patient who continue to have ambiguous diagnosis, while recognizing the limitations of chest CT to be cost, radiation exposure, delay, and lack of availability (particularly in resource limited environments).

\section{Lung ultrasonography for pulmonary edema versus ARDS} In the critically ill patient on mechanical ventilatory support, the differentiation of acute cardiogenic pulmonary edema (ACPE) from acute respiratory distress syndrome (ARDS) may be difficult [47, 48]. Lung ultrasonography is useful in distinguishing between the two entities. The presence of multiple bilateral B-lines on lung ultrasonography examination indicates the presence of an interstitial syndrome. Causes of this pattern of interstitial syndrome include such entities as acute cardiogenic pulmonary edema ACPE, pneumonia, interstitial pneumonitis, or ARDS. While signifying abnormality, B-lines are lacking in specificity; so, it is important to be able to identify the elements that increase their specificity. The pathophysiology of the underlying disease process determines the distribution of the B-lines and generates other 
ultrasound signs that are fundamental to the differential diagnosis. This becomes important when using lung ultrasonography to differentiate between ACPE, where the interstitial syndrome derives from an elevation of left atrial pressure; and ARDS, where parenchymal lung injury results in the interstitial syndrome detected with lung ultrasonography. The distinction between the two is a key differential point in managing the critically ill patient on mechanical ventilatory support with bilateral chest radiographic abnormalities that may be either from ACPE or ARDS.

With ACPE, B-lines result from elevation of left atrial pressure. With elevation of left atrial pressure, there is engorgement of venules within the interlobular septae that abut the visceral pleural surface. These are detected as B-lines with lung ultrasonography. There may be contribution from engorgement of interlobular lymphatics as well as accumulation of fluid within the alveolar compartment. In comparison, in ARDS B-lines result from primary lung injury, as they are formed by abnormalities adjacent to the visceral pleural surface that are derived directly from the lung injury.

The different pathophysiology in patients with ARDS and ACPE produces different pleuropulmonary ultrasound patterns with a different distribution of B-lines $[49,50]$. In patients with ARDS, lung ultrasonography is associated with characteristic patterns. In the nondependent lung fields, there are bilateral, non-homogeneously distributed B-lines. In some areas, B-lines are numerous; in others, they are coalescent, and between these two there are areas of normal lung (indicated by A-lines) configuring a non-homogeneous distribution of interstitial syndrome. In the posterior lung fields, B-lines are more homogeneous, showing the presence of coalescent B-lines that produce a "white lung" pattern. Areas of consolidation are often present in posterior fields, especially at the bases, with evidence of static or dynamic air bronchograms. "Lung sliding" is often reduced or absent in the areas of coalescent B-lines. The pleural line appears irregular, thickened and coarse with the presence of multiple small consolidations. Involvement of the pleural line is not homogeneous and follows the distribution and the degree of the interstitial syndrome pleural effusions is common but small. Lung ultrasonography findings are the same for primary and secondary ARDS.

The sonographic appearance of ACPE is characterized by interstitial syndrome homogeneously involving both anterior and posterior lung fields [49]. Anterior lung fields may be less affected, but "spared areas" are not observed, i.e., B-line distribution is homogeneous without interposed areas of normal aeration pattern (A-lines). The pleural line is smooth with normal lung sliding. Small consolidations can be found in some cases, particularly at the posterior bases. Pleural effusions are common and usually larger than in ARDS.

While lung ultrasonography is useful to distinguish between ARDS and APE, there are pitfalls. An occasional patient may have both ARDS and ACPE. It would be difficult to distinguish one from the other with lung ultrasonography alone. In this case, echocardiography is useful including both 2-D imaging and estimation of left atrial pressure using Doppler-based measurements. Barring these factors, lung ultrasonography is a useful tool to distinguish between ARDS and ACPE in the critically ill patient on mechanical ventilatory support with severe bilateral chest radiographic abnormality (Fig. 3; Table 1).

\section{Lung ultrasonography for evaluation of pulmonary embolism}

Lung ultrasonography may be used for the evaluation of PE in three different ways: as a stand-alone imaging method, in combination with other elements of critical care echocardiography, or in conjunction with pre-test probability.

As a stand-alone imaging method, lung ultrasonography is used to detect abnormalities that are associated with PE. Emboli in the pulmonary artery tree often create peripheral infarctions represented by regional loss of aeration due to hemorrhage and alveoli congestion. Lung ultrasonography can visualize these areas of abnormality when they are adjacent to the chest wall, even when they are limited to small areas of the lung periphery [51]. They appear as small consolidations that are usually wedge or round shaped, with sharp margins, and with a diameter larger than $5 \mathrm{~mm}$ measured on the pleural surface [52]. They are most often found in posterior lower lung zones and they can be detected both with the low-frequency phased array or curvilinear probes or with the high-frequency vascular probe of linear design. This finding has limited value, as it has only $74 \%$ sensitivity for the diagnosis of PE. Another limitation is that the finding may be time dependent; it is not clear how long the lesions take to appear following the occurrence of $\mathrm{PE}$. Prospective observational studies have demonstrated that a complete ultrasonography scan of the thorax in patients suspected for PE has the potential to aid in the diagnosis based on the detection of peripheral lesions with the characteristics of infarctions [51]. The first international consensus conference on point-of-care lung ultrasonography stated that it is an alternative to CT scan for the diagnosis of PE, when the latter is not available or contraindicated [4].

Lung ultrasonography, when integrated into a multiorgan ultrasonography evaluation, allows increased diagnostic efficiency compared to when it is used alone. This derives from the observation that when lung, cardiac and venous ultrasonography are combined for diagnostic 

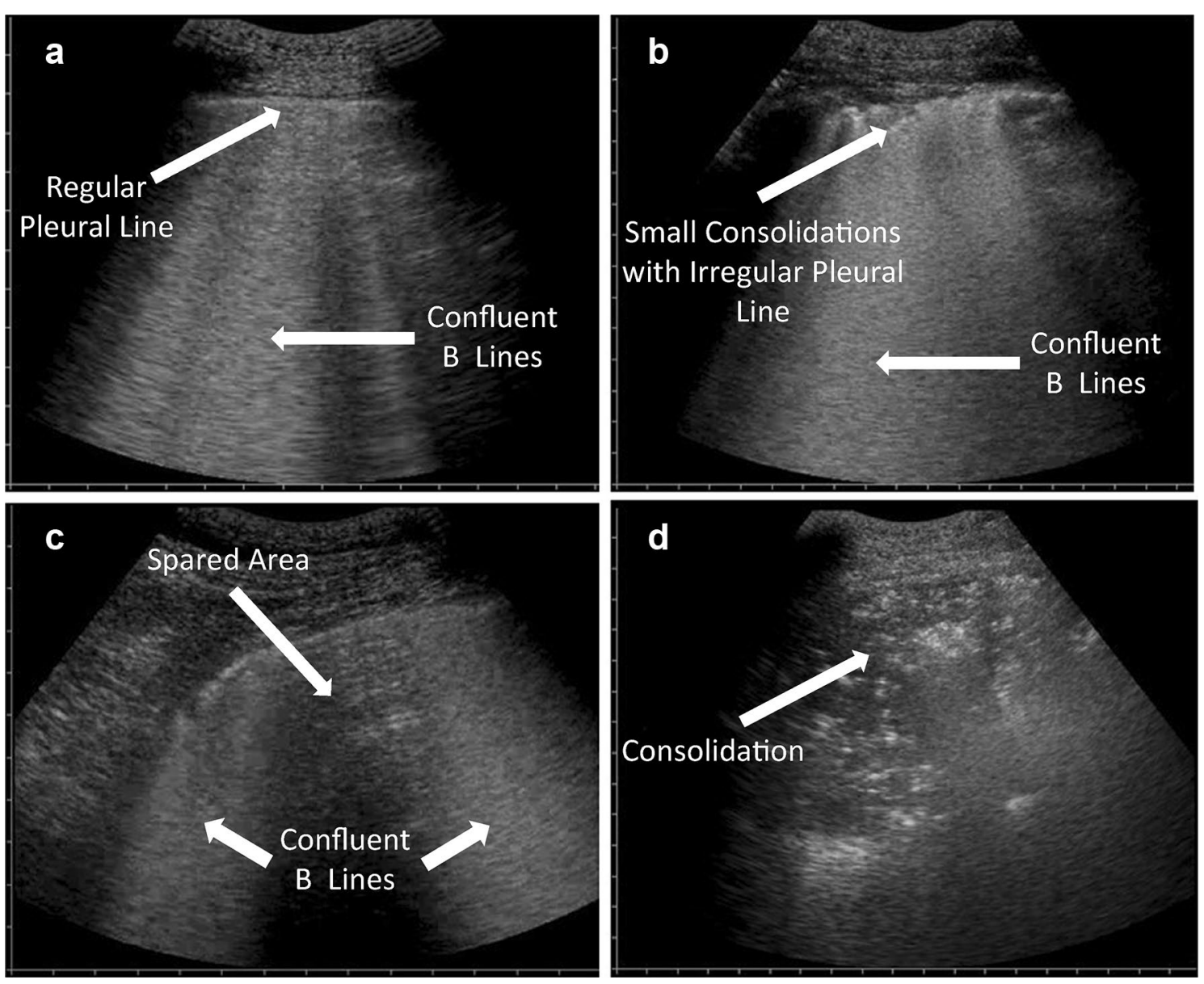

Fig. 3 Examples of lung ultrasonography relevant to differentiation of adult respiratory distress syndrome (ARDS) from acute cardiogenic pulmonary edema (ACPE). a Smooth pleural surface in association with confluent B-lines. This pattern is characteristic of ACPE. b Irregular pleural surface in association with small areas of consolidation. This pattern is characteristic of ARDS. $\mathbf{c} B$-lines with spared areas. This pattern is characteristic of ARDS. d Non-translobar consolidation. This pattern is characteristic of ARDS

workup, the results maintain good specificity for rendering diagnosis of PE. When considered alone, each single ultrasonography method has limitations in sensitivity, i.e., a limited potential for ruling out the disease when the ultrasonography test is negative. However, when considered in combination, the absence of lung ultrasonography findings consistent with pulmonary infarctions, the absence of right ventricle dilation, and the finding of fully compressible deep veins allow exclusion of PE with higher sensitivity [53]. Moreover, negative lung, cardiac and venous ultrasonography studies, together with the ultrasonography demonstration of alternatives to PE, such as pneumonia, pericardial effusion, pneumothorax, or pulmonary edema, increase the negative predictive value close to $100 \%$ [54].

Lung ultrasonography may be productively combined with pre-test risk scoring in the diagnostic workup for PE. Risk scoring is a fundamental step in the Bayesian approach to PE. It may allow the intensivist to rule out the disease with safety or it may indicate the necessity of a second level diagnostic such as CT pulmonary angiography [55]. However, risk scoring is based on clinical signs and judgments that have low specificity and that are subjective in nature [56]. When venous and lung ultrasonography are used to supplement risk scoring with an objective diagnosis of deep venous thrombosis and pulmonary infarctions or alternative pleuropulmonary diseases, the efficiency of risk scoring significantly increases; and a high percentage of negative CT studies can be avoided [57].

\section{Lung ultrasonography for evaluation of pneumonia and interstitial processes}

Lung ultrasonography has the utility for the diagnosis of pneumonia. Like CXR and chest CT, the diagnosis of pneumonia is never predicated solely on the results of 
imaging alone but requires integration of the results of thoracic imaging (regardless of modality) with the history, the physical examination, and laboratory results. This being a given, lung ultrasonography is superior to chest radiography for the identification of findings consistent with pneumonia with chest $\mathrm{CT}$ as the "gold standard" and has a diagnostic accuracy that approaches chest CT $[58,59]$. There are eight metanalyses of lung ultrasonography in community-acquired pneumonia with four using chest CT as reference method. The pooled sensitivity $(85-96 \%)$ and specificity $(80-96 \%)$ are very high and accuracy more than 90\% [60]. Lung ultrasonography may largely replace chest radiography for the diagnosis of acute community-acquired pneumonia [61].

The characteristic feature of pneumonia on lung ultrasonography is the finding of consolidation. The areas of consolidation may be in the millimeter range limited to an area immediately below the pleural line. Larger areas of consolidation may be either non-translobar or translobar (consolidation occupying the entire lobe). The area of involvement may be focal or multifocal. A mobile air bronchogram is suggestive of pneumonia [62]. These appear as punctate or linear hyperechoic structures within the area of consolidation that move in phase with the respiratory cycle.

On color Doppler ultrasonography, consolidation has a typical appearance: circulation is uniformly branched and vessels have a regular course [63]. With contrastenhanced ultrasonography (CEUS), there is a short washin period and an intensive enhancement. CEUS is useful in differentiating inflammatory from embolic lung consolidation, especially in patients with indeterminate chest CT findings [64]. CEUS may be used in ambiguous cases to diagnose lung abscesses within pneumonia, as CEUS is superior to 2-D ultrasonography or CXR for this application [65].

Pneumonia may be associated with B-lines that may be sub-segmental, segmental, lobar, multifocal, or generalized distribution depending on the severity of disease. This emphasizes the point that lung ultrasonography results are integrated into the whole clinical picture, as there are other disease processes that result in B-lines. Lung ultrasonography is useful in tracking the improvement of pneumonia including ventilator-associated pneumonia [66]. When pneumonia is in the healing phase, the infiltrated lung tissue is increasingly aerated. With re-aeration, air within the affected lung gives rise to reflection and reverberation artefacts within affected lung. The pneumonia recedes on the lung ultrasonography image and appears smaller than on chest radiograph.

The margin of pneumonia is characterized by an irregular, serrated and somewhat blurred shape with comet tail artefacts. In early stages a fluid bronchogram may be visible, characterized by anechoic/hypoechoic branched tubular structures in the course of the bronchial tree. A persistent fluid bronchogram arouses suspicion of poststenotic pneumonia and that may require bronchoscopic investigation $[4,67]$. Bacterial pneumonias may undergo necrosis and form abscesses that appear as round or oval and largely anechoic lesions within the consolidated lung. The lung abscess may have an echogenic margin and contain an air artefact within. If a patient does not respond to treatment with antibiotics, the pathogen can be acquired by means of ultrasonography-guided aspiration [68].

Interstitial lung diseases are readily detected with lung ultrasonography, as the process frequently extends to the subpleural area [7]. This yields a pattern of visceral pleural irregularity with a non-homogeneous distribution of B-lines. The visceral pleural interface is disrupted and fragmented. Small consolidations may occur with associated comet tails in a non-homogeneous distribution. The presence of B-lines may be a valuable marker of disease severity in patients with interstitial pneumonia that correlates to the extent of the reticular pattern on chest high-resolution computed tomography [69].

\section{Lung ultrasonography for management of the patient on mechanical ventilatory support}

Lung ultrasonography is useful for the management of critically ill patients on mechanical ventilatory support [6]. Progressive loss of lung aeration is associated with different lung ultrasonography patterns, so an ultrasonography-based scoring system has been proposed to assess lung tissue aeration. The lung ultrasonography score (LUS) (Table 2) allows pseudo-quantification of regional loss of aeration: each step increase of this score is associated with a significant increase of lung tissue density as assessed by quantitative CT scan [70]. By grading anterior, lateral and posterior regions of the two lungs, a global LUS can be computed: it quantifies the overall loss of aeration, with strong correlation with lung weight and extra-vascular lung water [70, 71]. Thus, the global LUS may provide a reliable and objective assessment of the severity of acute respiratory failure. A daily lung ultrasonography assessment that includes the LUS can be easily and quickly performed at the bedside [72] and can be used to monitor the evolution of the respiratory disease [73]; this is particularly useful in severely affected lungs with poorly informative chest X-ray and high-risk transportation to $\mathrm{CT}$ scan.

Lung ultrasonography may also help to set PEEP in mechanically ventilated patients. Patients with focal loss of aeration at CT scan are poor PEEP responders, whereas those with a more diffuse disease may positively respond to PEEP [74]. Disease morphology (focal vs. diffuse) can be conveniently assessed with ultrasound 
Table 2 Lung ultrasound score and corresponding ultrasound patterns

\begin{tabular}{|c|c|c|c|}
\hline Points & Degrees of lung aeration & Patterns & \\
\hline 0 point & Normal aeration & $\begin{array}{l}\text { Horizontal } A \text {-lines } \\
\text { (or no more than two B- } \\
\text { lines) }\end{array}$ & $\therefore$ \\
\hline 1 point & $\begin{array}{l}\text { Moderate loss of } \\
\text { aeration }\end{array}$ & $\begin{array}{l}\text { Multiple B-lines, } \\
\text { either regularly spaced ( } 7 \\
\text { mm apart), or irregularly } \\
\text { spaced and even } \\
\text { coalescent but only } \\
\text { visible in a limited area of } \\
\text { the intercostal space }\end{array}$ & 8 \\
\hline 2 points & Severe loss of aeration & $\begin{array}{l}\text { Multiple coalescent B- } \\
\text { lines, in prevalent areas } \\
\text { of the intercostal spaces } \\
\text { and observed in one or } \\
\text { several intercostal } \\
\text { spaces }\end{array}$ & $=$ \\
\hline 3 points & $\begin{array}{l}\text { Complete loss of } \\
\text { aeration }\end{array}$ & $\begin{array}{l}\text { Lung consolidation, } \\
\text { with or without air } \\
\text { bronchograms }\end{array}$ & \\
\hline
\end{tabular}

at the bedside [75]. A re-aeration LUS can be computed to assess the PEEP-induced gain in end-expiratory lung volume [75]; this does not strictly correspond to recruitment of previously collapsed lung tissue, as a substantial proportion of the volume gain pertains to already, although poorly, aerated lung tissue. A weakness of lung ultrasonography in ARDS is that it cannot detect overdistention [75].

Lung ultrasonography also provides dynamic information: a severely decreased or abolished lung sliding suggests impaired regional ventilation. This can be associated with high PEEP setting [76], thus suggesting PEEP-induced hyperinflation in non-dependent lung regions, especially if lung sliding reappears when PEEP is decreased [48].

Three studies have examined lung ultrasonography in patient subjected to prone position for severe ARDS with conflicting results. One study found that a normal LUS pattern of both anterobasal lung regions in supine position predicts a significant improvement in oxygenation function [77], whereas another study found that oxygenation response after prone position was not correlated with a specific lung ultrasonography pattern [78]. Another study indicated that improvement in aeration detected by lung ultrasonography upon proning predicts improvement in oxygenation by day 7 and improved survival [79]. The LUS may guide fluid management in ARDS septic patients: in this setting, the LUS score variations are more sensitive than oxygenation in early detection of lung aeration deterioration due to fluid loading [80].

Lung ultrasonography is useful in identifying pneumonia in the patient on mechanical ventilatory support [81, 82 ] and it can then be used to assess antibiotic-induced re-aeration and redirect therapy in non-resolving cases [66]. Lung ultrasonography findings such as lobar and subpleural consolidations are very sensitive but poorly specific in suggesting ventilator-associated pneumonia; 
a dynamic linear-arborescent air bronchogram is a more specific sign [83]. Ultrasonography findings also require integration into clinical assessment. Combining ultrasonography findings with laboratory testing such as Gram stain of sputum or measurement of procalcitonin can improve the diagnostic accuracy $[83,84]$. Ultrasound features of lung consolidations may help identifying the cause and guide treatments. If air bronchogram is absent or static in the consolidated lung, air transit is prevented in the corresponding airway and de-obstructive bronchoscopy may improve downstream aeration. Instead, a linear-arborescent dynamic air bronchogram strongly suggests ventilator-associated pneumonia; thus, microbiological sampling and empiric antibiotic treatment may be indicated [81]. Color Doppler can identify a luxuriant perfusion of a consolidated lung region, confirming the presence of intrapulmonary shunt and suggesting a significant contribution of the visualized region to patient's hypoxemia [84].

The LUS can help in the weaning from mechanical ventilation. After a successful weaning trial, patients who will experience post-extubation distress have a lower LUS than those successfully extubated [85]. Similarly, lung ultrasonography assessment at ICU admission after major abdominal surgery may predict the need for prolonged postoperative respiratory support [86]. Combining lung ultrasonography with echocardiography may be helpful in determining the cause for weaning failure [87, 88], as does ultrasonography assessment of diaphragm function [89]. Lung ultrasonography has utility for the detection of weaning-induced pulmonary edema [90].

\section{Conclusion}

Mastery of lung and pleural ultrasonography allows the intensivist to rapidly diagnose and guide the management of a wide variety of disease processes that are common features of critical illness. Its ease of use, rapidity, repeatability, and reliability make thoracic ultrasonography the "go to" modality for imaging the lung and pleura in an efficient, cost effective, and safe manner. Thoracic ultrasonography can largely replace CXR in the ICU and may reduce the need of for chest $\mathrm{CT}$ in critical care practice. It is best combined with other components of CCUS at point of care to result in a comprehensive evaluation of the critically ill patient.

\section{Electronic supplementary material}

The online version of this article (https://doi.org/10.1007/s00134-019-05725-8) contains supplementary material, which is available to authorized users.

\footnotetext{
Author details

${ }^{1}$ Division of Pulmonary, Critical Care and Sleep Medicine, Northwell Health, Zucker School of Medicine at Hofstra/Northwell, New Hyde Park, Hempstead NY 11549, USA. ${ }^{2}$ Department of Emergency Medicine, Latisana Hospital, 33053 Latisana, Italy. ${ }^{3}$ Division of Pulmonary, Critical Care, and Sleep Medicine,
}

Johns Hopkins Hospital, Sheikh Zayed Tower, Suite 7-125, 1800 Orleans Street, Baltimore, MD 21287, USA. ${ }^{4} 3$ Praxis for Internal Medicine, Bahnhofstraße 16, 6830 Rankweil, Austria. ${ }^{5} 7$ Medical Intensive Care Unit, Assistance Publique-Hôpitaux de Paris, University Hospital Saint-Antoine, Paris, France. ${ }^{6} 8$ INSERM U 1136, Institut Pierre-Louis d'Epidémiologie et de Santé Publique, 75012 Paris, France. ${ }^{7} 9$ Sorbonne University, UPMC Univ Paris 06, Paris, France. ${ }^{8}$ Anesthesia and Intensive Care, IRCCS Policlinico San Matteo, University of Pavia, Pavia, Italy. ${ }^{9}$ Department of Emergency Medicine, San Luigi Gonzaga University Hospital, Orbassano, 10043 Turin, Italy. ${ }^{10}$ Emergency Department, Careggi University Hospital, Largo Brambilla 3, 50134 Florence, Italy.

\section{Compliance with ethical standards}

\section{Conflicts of interest}

The authors do not declare any conflict of interest.

\section{Publisher's Note}

Springer Nature remains neutral with regard to jurisdictional claims in published maps and institutional affiliations.

Received: 7 May 2019 Accepted: 30 July 2019

Published online: 15 August 2019

\section{References}

1. Brogi E, Bignami E, Sidoti A, Shawar M, Gargani L, Vetrugno L, Volpicelli $G$, Forfori $F(2017)$ Could the use of bedside lung ultrasound reduce the number of chest $\mathrm{x}$-rays in the intensive care unit? Cardiovasc Ultrasound 15:23

2. Oks M, Cleven KL, Cardenas-Garcia J, Schaub JA, Koenig S, Cohen Rl, Mayo PH, Narasimhan M (2014) The effect of point-of-care ultrasonography on imaging studies in the medical ICU: a comparative study. Chest 146:1574-1577

3. Lichtenstein DA (2015) Lung ultrasound in the critically ill. Springer, New York

4. Volpicelli G, Elbarbary M, Blaivas M, Lichtenstein DA, Mathis G, Kirkpatrick AW, Melniker L, Gargani L, Noble VE, Via G, Dean A, Tsung JW, Soldati G, Copetti R, Bouhemad B, Reissig A, Agricola E, Rouby JJ, Arbelot C, Liteplo A, Sargsyan A, Silva F, Hoppmann R, Breitkreutz R, Seibel A, Neri L, Storti E, Petrovic T, International Liaison Committee on Lung Ultrasound (ILC-LUS) for International Consensus Conference on Lung Ultrasound (ICC-LUS) (2012) International evidence-based recommendations for point-of-care lung ultrasound. Intensive Care Med 38:577-591

5. Mathis G (2017) Chest sonography, 4th edn. Springer, Berlin, pp 51-59

6. Mojoli F, Bouhemad B, Mongodi S, Lichtenstein D (2019) Lung ultrasound for critically ill patients. Am J Respir Crit Care Med 199:701-714

7. Dietrich CF, Mathis G, Blaivas M, Volpicelli G, Seibel A, Wastl D, Atkinson NS, Cui XW, Fan M, Yi D (2016) Lung B-line artefacts and their use. J Thorac Dis 8:1356-1365

8. Lichtenstein D, Mezière G (2008) Relevance of lung ultrasound in the diagnosis of acute respiratory failure: the BLUE protocol. Chest 134:117-125

9. Lichtenstein DA (2014) Lung ultrasound in the critically ill. Ann Intensive Care 4:1-12

10. Lichtenstein D, Mezière G (2011) The BLUE-points: three standardized points used in the BLUE-protocol for ultrasound assessment of the lung in acute respiratory failure. Crit Ultrasound J 3:109-110

11. Cholley B (2011) International expert statement on training standards for critical care ultrasonography expert round table on ultrasound in ICU. Intensive Care Med 37:1077-1083

12. Alrajab S, Youssef AM, Akkus NI, Caldito G (2013) Pleural ultrasonography versus chest radiography for the diagnosis of pneumothorax: review of the literature and meta-analysis. Crit Care 17:R208

13. Avila J, Smith B, Mead T, Jurma D, Dawson M, Mallin M, Dugan A (2018) Does the addition of $\mathrm{M}$-mode to $\mathrm{B}$-mode ultrasound increase the accuracy of identification of lung sliding in traumatic pneumothoraces? J Ultrasound Med 37:2681-2687

14. Lichtenstein DA, Menu Y (1995) A bedside ultrasound sign ruling out pneumothorax in the critically ill: lung sliding. Chest 108:1345-1348 
15. Lichtenstein D, Mezière G, Biderman P, Gepner A (2000) The lung point: an ultrasound sign specific to pneumothorax. Intensive Care Med 26:1434-1440

16. Volpicelli G, Boero E, Sverzellati N, Cardinale L, Busso M, Boccuzzi F, Tullio M, Lamorte A, Stefanone V, Ferrari G, Veltri A, Frascisco MF (2014) Semiquantification of pneumothorax volume by lung ultrasound. Intensive Care Med 40:1460-1467

17. Lichtenstein DA, Lascols N, Prin S, Mezière G (2003) The "lung pulse" an early ultrasound sign of complete atelectasis. Intensive Care Med 29:2187-2192

18. Lichtenstein D, Meziere G, Biderman P, Gepner A (1999) The comet-tail artifact: an ultrasound sign ruling out pneumothorax. Intensive Care Med 25:383-388

19. Abbasi S, Farsi D, Hafezimoghadam P, Fathi M, Zare MA (2013) Accuracy of emergency physician-performed ultrasound in detecting traumatic pneumothorax after a 2-h training course. Eur J Emerg Med 20:173-177

20. Galbois A, Ait-Oufella H, Baudel JL, Kofman T, Bottero J, Viennot S, Rabate C, Jabbouri S, Bouzeman A, Guidet B, Offenstadt G, Maury E (2010) Pleural ultrasound compared with chest radiographic detection of pneumothorax resolution after drainage. Chest 138:648-655

21. Soult MC, Collins JN, Novosel TJ, Weireter LJ, Britt LD (2014) Thoracic ultrasound can predict safe removal of thoracostomy tubes. J Trauma Acute Care Surg 77:256-261

22. Mattison LE, Coppage L, Alderman DF, Herlong JO, Sahn SA (1997) Pleural effusions in the medical ICU: prevalence, causes, and clinical implications. Chest 111:1018-1023

23. Goligher EC, Leis JA, Fowler RA, Pinto R, Adhikari NK, Ferguson N (2011) Utility and safety of draining pleural effusions in mechanically ventilated patients: a systematic review and meta-analysis. Crit Care 15:R46

24. Yu CJ, Yang PC, Chang DB, Luh KT (1992) Diagnostic and therapeutic use of chest sonography: value in critically ill patients. AJR Am J Roentgenol 159:695-701

25. Kohan JM, Poe RH, Israel RH et al (1986) Value of chest ultrasonography versus decubitus roentgenography for thoracentesis. Am Rev Respir Dis 133:1124-1126

26. Balik M, Plasil P, Waldauf $P$ et al (2006) Ultrasound estimation of volume of pleural fluid in mechanically ventilated patients. Intensive Care Med 32:318-321

27. Teichgraber UK, Hackbarth J (2018) Sonographic bedside quantification of pleural effusion compared to computed tomography volumetry in ICU patients. Ultrasound Int Open 4:E131-E135

28. Vignon P, Chastagner C, Berkane V, Chardac E, François B, Normand S, Bonnivard M, Clavel M, Pichon N, Preux PM, Maubon A, Gastinne H (2005) Quantitative assessment of pleural effusion in critically ill patients by means of ultrasonography. Crit Care Med 33:1757-1763

29. Havelock T, Teoh R, Laws D, Gleeson F (2010) Pleural procedures and thoracic ultrasound: British Thoracic Society pleural disease guideline 2010. Thorax 65:161-i76

30. Razazi K, Boissier F, Neuville M, Jochmans S, Tchir M, May F, de Prost N, Brun-Buisson C, Carteaux G, Mekontso Dessap A (2018) Pleural effusion during weaning from mechanical ventilation: a prospective observational multicenter study. Ann Intensive Care 8:103

31. Feller-Kopman D (2006) Ultrasound-guided thoracentesis. Chest 129:1709-1714

32. Millington SJ, Koenig S (2018) Better with ultrasound: pleural procedures in critically ill patients. Chest 153:224-232

33. Gordon CE, Feller-Kopman D, Balk EM, Smetana GW (2010) Pneumothorax following thoracentesis: a systematic review and meta-analysis. Arch Int Med 170:332-339

34. Mayo PH, Goltz HR, Tafreshi M, Doelken P (2004) Safety of ultrasoundguided thoracentesis in patients receiving mechanical ventilation. Chest 125:1059-1062

35. Kanai M, Sekiguchi H (2015) Avoiding vessel laceration in thoracentesis: a role of vascular ultrasound with color Doppler. Chest 147:e5-e7

36. Yang PC, Luh KT, Chang DB, Wu HD, Yu CJ, Kuo SH (1992) Value of sonography in determining the nature of pleural effusion: analysis of 320 cases. AJR Am J Roentgenol 159:29-33

37. McLoud TC, Flower CD (1991) Imaging the pleura: sonography, CT, and MR imaging. AJR Am J Roentgenol 156(6):1145-1153

38. Lichtenstein D, Goldstein I, Mourgeon E, Cluzel P, Grenier P, Rouby JJ (2004) Comparative diagnostic performances of auscultation, chest radiography, and lung ultrasonography in acute respiratory distress syndrome. Anesthesiology 100:9-15

39. Xirouchaki N, Magkanas E, Vaporidi K, Kondili E, Plataki M, Patrianakos A, Akoumianaki E, Georgopoulos D (2011) Lung ultrasound in critically ill patients: comparison with bedside chest radiography. Intensive Care Med 37:1488-1493

40. Pivetta E, Goffi A, Nazerian P, Castagno D, Tozzetti C, Tizzani P, Tizzani M, Porrino G, Ferreri E, Busso V, Morello F, Paglieri C, Masoero M, Cassine E, Bovaro F, Grifoni S, Maule MM, Lupia E, Study Group on Lung Ultrasound from the Molinette and Careggi Hospitals (2019) Lung ultrasound integrated with clinical assessment for the diagnosis of acute decompensated heart failure in the emergency department: a randomized controlled trial. Eur J Heart Fail. (Epub ahead of print)

41. Pivetta E, Goffi A, Lupia E, Tizzani M, Porrino G, Ferreri E, Volpicelli G, Balzaretti P, Banderali A, lacobucci A, Locatelli S, Casoli G, Stone MB, Maule MM, Baldi I, Merletti F, Cibinel GA, Baron P, Battista S, Buonafede G, Busso V, Conterno A, Del Rizzo P, Ferrera P, Pecetto PF, Moiraghi C, Morello F, Steri F, Ciccone G, Calasso C, Caserta MA, Civita M, Condo' C, D'Alessandro V, Del Colle S, Ferrero S, Griot G, Laurita E, Lazzero A, Lo Curto F, Michelazzo M, Nicosia V, Palmari N, Ricchiardi A, Rolfo A, Rostagno R, Bar F, Boero E, Frascisco M, Micossi I, Mussa A, Stefanone V, Agricola R, Cordero G, Corradi F, Runzo C, Soragna A, Sciullo D, Vercillo D, Allione A, Artana N, Corsini F, Dutto L, Lauria G, Morgillo T, Tartaglino B, Bergandi D, Cassetta I, Masera C, Garrone M, Ghiselli G, Ausiello L, Barutta L, Bernardi E, Bono A, Forno D, Lamorte A, Lison D, Lorenzati B, Maggio E, Masi I, Maggiorotto M, Novelli G, Panero F, Perotto M, Ravazzoli M, Saglio E, Soardo F, Tizzani A, Tizzani P, Tullio M, Ulla M, Romagnoli E, SIMEU Group for Lung Ultrasound in the Emergency Department in Piedmont (2015) Lung ultrasoundimplemented diagnosis of acute decompensated heart failure in the ED: a SIMEU multicenter study. Chest 148:202-210

42. Laursen CB, Sloth E, Lassen AT, dePont Christensen R, Lambrechtsen J, Madsen PH, Henriksen DP, Davidsen JR, Rasmussen F (2014) Point-ofcare ultrasonography in patients admitted with respiratory symptoms: a single-blind, randomised controlled trial. Lancet Respir Med 2:638-646

43. Goffi A, Pivetta E, Lupia E, Porrino G, Civita M, Laurita E, Griot G, Casoli G, Cibinel $G$ (2013) Has lung ultrasound an impact on the management of patients with acute dyspnea in the emergency department? Crit Care 17:R180

44. Zanobetti M, Poggioni C, Pini R (2011) Can chest ultrasonography replace standard chest radiography for evaluation of acute dyspnea in the ED? Chest 139:1140-1147

45. Zanobetti M, Scorpiniti M, Gigli C, Nazerian P, Vanni S, Innocenti F, Stefanone VT, Savinelli C, Coppa A, Bigiarini S, Caldi F, Tassinari I, Conti A, Grifoni S, Pini R (2017) Point-of-care ultrasonography for evaluation of acute dyspnea in the ED. Chest 151:1295-1301

46. Bekgoz B, Kilicaslan I, Bildik F, Keles A, Demircan A, Hakoglu O, Coskun G, Demir HA (2019) BLUE protocol ultrasonography in emergency department patients presenting with acute dyspnea. Am J Emerg Med. pii: S0735-6757(19)30112-3. https://doi.org/10.1016/j.ajem.2019.02.028. (Epub ahead of print)

47. Ranieri VM, Rubenfeld GD, Thompson BT, Ferguson ND, Caldwell E, Fan E, Camporota L, Slutsky AS (2012) Acute respiratory distress syndrome: the Berlin definition. ARDS definition task force. JAMA 307:2526-2533

48. Pesenti A, Musch G, Lichtenstein D, Mojoli F, Amato MBP, Cinnella G, Gattinoni L, Quintel M (2016) Imaging in acute respiratory distress syndrome. Intensive Care Med 42:686-698

49. Copetti R, Soldati G, Copetti P (2008) Chest sonography: a useful tool to differentiate acute cardiogenic pulmonary edema from acute respiratory distress syndrome. Cardiovasc Ultrasound 6:16

50. Al Deeb M, Barbic S, Featherstone R, Dankoff J, Barbic D (2014) Point-ofcare ultrasonography for the diagnosis of acute cardiogenic pulmonary edema in patients presenting with acute dyspnea: a systematic review and meta-analysis. Acad Emerg Med 21:843-852

51. Mathis G, Blank W, Reissig A, Lechleitner P, Reuss J, Schuler A, Beckh S (2005) Thoracic ultrasonography for diagnosing PE: a prospective multicenter study of 352 patients. Chest 128:1531-1538

52. Volpicelli G (2013) Lung sonography. J Ultrason Med 32:165-171

53. Nazerian P, Vanni S, Volpicelli G, Gigli C, Zanobetti M, Bartolucci M, Ciavattone A, Lamorte A, Veltri A, Fabbri A, Grifoni S (2014) Accuracy of point-ofcare multiorgan ultrasonography for the diagnosis of pulmonary embolism. Chest 145:950-957 
54. Koenig S, Chandra S, Alaverdian A, Dibello C, Mayo PH, Narasimhan M (2014) Ultrasonography assessment of $\mathrm{PE}$ in patients receiving CT pulmonary angiography. Chest 145:818-823

55. Wells PS, Anderson DR, Rodger M, Stiell I, Dreyer JF, Barnes D, Forgie M, Kovacs G, Ward J, Kovacs MJ (2001) Excluding PE at the bedside without diagnostic imaging: management of patients with suspected PE presenting to the emergency department by using a simple clinical model and d-dimer. Ann Intern Med 135:98-107

56. Konstantinides SV, Torbicki A, Agnelli G et al (2014) 2014 ESC guidelines on the diagnosis and management of acute PE. Eur Heart J 35:3033-3069

57. Nazerian P, Volpicelli G, Gigli C, Becattini C, Sferrazza Papa GF, Grifoni S, Vanni S, Ultrasound Wells Study Group (2017) Diagnostic performance of wells score combined with point-of-care lung and venous ultrasonography in suspected PE. Acad Emerg Med 24:270-280

58. Nazerian P, Volpicelli G, Vanni S, Gigli C, Betti L, Bartolucci M, Zanobetti M, Ermini FR, lannello C, Grifoni S (2015) Accuracy of lung ultrasound for the diagnosis of consolidations when compared to chest computed tomography. Am J Emerg Med 33:620-625

59. Reissig A, Copetti R, Mathis G, Mempel C, Schuler A, Zechner P, Aliberti S, Neumann R, Kroegel C, Hoyer H (2012) Lung ultrasound in the diagnosis and follow-up of community-acquired pneumonia: a prospective, multicenter, diagnostic accuracy study. Chest 142:965-972

60. Ye X, Xiao H, Chen B, Zhang S (2015) Accuracy of lung ultrasonography versus chest radiography for the diagnosis of adult community-acquired pneumonia. PLoS One 10:e0130066

61. Mathis G (2018) Pneumonia: does ultrasonography replace chest $X$-ray? Ersetzt der Praxis 107:1-5

62. Lichtenstein D, Mezière G, Seitz J (2009) The dynamic air bronchogram. A lung ultrasound sign of alveolar consolidation ruling out atelectasis. Chest 135:1421-1425

63. Xirouchaki N, Pediaditis M, Proklou A, Georgopoulos D (2017) Tree-like colour Doppler in diagnosing pneumonia in critically ill: a picture is worth a thousand words. Intensive Care Med 44:494-495

64. Goerg C (2007) Transcutaneous contrast-enhanced sonography of pleuralbased pulmonary lesions. EJR 64:213-221

65. Sidhu PS, Cantisani V, Dietrich CF, Gilja OH, Saftoiu A, Bartels E, Bertolotto M, Calliada F, Clevert DA, Cosgrove D, Deganello A, D'Onofrio M, Drudi FM, Freeman S, Harvey C, Jenssen C, Jung EM, Klauser AS, Lassau N, Meloni MF, Leen E, Nicolau C, Nolsoe C, Piscaglia F, Prada F, Prosch H, Radzina M, Savell L, Weskott HP, Wijkstra H (2012) The EFSUMB guidelines and recommendations on the clinical practice of contrast enhanced ultrasonography (CEUS): update 2011 on non-hepatic applications. Ultraschall in Med 33:33-59

66. Bouhemad B, Liu ZH, Arbelot C, Zhang M, Ferarri F, Le-Guen M, Girard M, Lu Q, Rouby JJ (2010) Ultrasound assessment of antibiotic-induced pulmonary reaeration in ventilator-associated pneumonia. Crit Care Med 38:84-92

67. Reissig A, Copetti R (2014) Lung ultrasonography in community-acquired pneumonia and in interstitial lung diseases. Respiration 87:179-189

68. Yang PC, Luh KT, Lee YC (1991) Lung abscesses: ultrasonography and ultrasonography-guided transthoracic aspiration. Radiology 180:171-175

69. Asano M, Watanabe H, Sato Ket al (2018) Validity of ultrasonography lung comets for assessment of the severity of interstitial pneumonia. J Ultrason Med 37:1523-1531

70. Chiumello D, Mongodi S, Algieri I, Vergani GL, Orlando A, Via G, Crimella F, Cressoni M, Mojoli F (2018) Assessment of lung aeration and recruitment by CT scan and ultrasound in ARDS patients. Crit Care Med 46:1761-1768

71. Zhao Z, Jiang $L$, Xi X, Jiang Q, Zhu B, Wang M, Xing J, Zhang D (2015) Prognostic value of extravascular lung water assessed with lung ultrasound score by chest sonography in patients with acute respiratory distress syndrome. BMC Pulm Med 15:98

72. Rouby JJ, Arbelot C, Gao Y, Zhang M, LV J, An Y, Wang C, Bin D, Barbas CSV, Dexheimer Neto FL, Prior Caltabeloti F, Lima E, Cebey A, Perbet S, Constantin JM, APECHO study group (2018) Training for lung ultrasound score measurement in critically ill patients. Am J Respir Crit Care Med. https://doi. org/10.1164/rccm.201802-0227le. (Epub ahead of print)

73. Mongodi S, Pozzi M, Orlando A, Bouhemad B, Stella A, Tavazzi G, Via G, lotti GA, Mojoli F (2018) Lung ultrasound for daily monitoring of ARDS patients on extracorporeal membrane oxygenation: preliminary experience. Intensive Care Med 44:123-124
74. Constantin JM, Grasso S, Chanques G, Aufort S, Futier E, Sebbane M, Jung B, Gallix B, Bazin JE, Rouby JJ, Jaber S (2010) Lung morphology predicts response to recruitment maneuver in patients with acute respiratory distress syndrome. Crit Care Med 38:1108-1117

75. Bouhemad B, Brisson H, Le-Guen M, Arbelot C, Lu Q, Rouby JJ (2011) Bedside ultrasound assessment of positive end-expiratory pressure-induced lung recruitment. Am J Respir Crit Care Med 183:341-347

76. Markota A, Golub J, Stožer A, Fluher J, Prosen G, Bergauer A, Svenšek F, Sinkovič A (2016) Absence of lung sliding is not a reliable sign of pneumothorax in patients with high positive end-expiratory pressure. Am J Emerg Med 34:2034-2036

77. Prat G, Guinard S, Bizien N, Nowak E, Tonnelier JM, Alavi Z, Renault A, Boles JM, L’Her E (2016) Can lung ultrasonography predict prone positioning response in acute respiratory distress syndrome patients? J Crit Care 32:36-41

78. Haddam M, Zieleskiewicz L, Perbet S, Baldovini A, Guervilly C, Arbelot C, Noel A, Vigne C, Hammad E, Antonini F, Lehingue S, Peytel E, Lu Q, Bouhemad B, Golmard JL, Langeron O, Martin C, Muller L, Rouby JJ, Constantin JM, Papazian L, Leone M, CAR'Echo Collaborative Network, AzuRea Collaborative Network (2016) Lung ultrasonography for assessment of oxygenation response to prone position ventilation in ARDS. Intensive Care Med 42:1546-1556

79. Wang XT, Ding X, Zhang HM, Chen H, Su LX, Liu DW, Chinese Critical Ultrasound Study Group (CCUSG) (2016) Lung ultrasound can be used to predict the potential of prone positioning and assess prognosis in patients with acute respiratory distress syndrome. Crit Care 20:385

80. Caltabeloti F, Monsel A, Arbelot C, Brisson H, Lu Q, Gu WJ, Zhou GJ, Auler JO, Rouby JJ (2014) Early fluid loading in acute respiratory distress syndrome with septic shock deteriorates lung aeration without impairing arterial oxygenation: a lung ultrasound observational study. Crit Care 18:R91

81. Mongodi S, Via G, Girard M, Rouquette I, Misset B, Braschi A, Mojoli F, Bouhemad B (2016) Lung ultrasound for early diagnosis of ventilator-associated pneumonia. Chest 149:969-980

82. Staub $\sqcup$, Biscaro RR, Maurici R (2018) Accuracy and applications of lung ultrasound to diagnose ventilator-associated pneumonia: a systematic review. J Intensive Care Med 33:447-455

83. Zhou J, Song J, Gong S, Hu W, Wang M, Xiao A, Zhang C, Dong Z. (2019) Lung ultrasound combined with procalcitonin for a diagnosis of ventilatorassociated pneumonia. Respir Care. pii: 06377. https://doi.org/10.4187/respc are.06377. (Epub ahead of print)

84. Mongodi S, Bouhemad B, lotti GA, Mojoli F (2016) An ultrasonographic sign of intrapulmonary shunt. Intensive Care Med 42:912-913

85. Soummer A, Perbet S, Brisson H, Arbelot C, Constantin JM, Lu Q, Rouby JJ, Lung Ultrasound Study Group (2012) Ultrasound assessment of lung aeration loss during a successful weaning trial predicts postextubation distress. Crit Care Med 40:2064-2072

86. Dransart-Rayé O, Roldi E, Zieleskiewicz L, Guinot PG, Mojoli F, Mongodi S, Bouhemad B (2019) Lung ultrasound for early diagnosis of postoperative need for ventilation support: a prospective observational study. Anaesthesia (provisionally accepted)

87. Mongodi S, Via G, Bouhemad B, Storti E, Mojoli F, Braschi A (2013) Usefulness of combined bedside lung ultrasound and echocardiography to assess weaning failure from mechanical ventilation: a suggestive case. Crit Care Med 41:e182-e185

88. Mayo P, Volpicelli G, Lerolle N, Schreiber A, Doelken P, Vieillard-Baron A (2016) Ultrasonography evaluation during the weaning process: the heart, the diaphragm, the pleura and the lung. Intensive Care Med 42:1107-1117

89. Tenza-Lozano E, Llamas-Alvarez A, Jaimez-Navarro E, Fernàndez-Sanchez J (2018) Lung and diaphragm ultrasound as predictors of success in weaning from mechanical ventilation. Crit Ultrasound J 10:12

90. Ferré A, Guillot M, Lichtenstein D, Mezière G, Richard C, Teboul JL, Monnet X (2019) Lung ultrasound allows the diagnosis of weaning-induced pulmonary oedema. Intensive Care Med 45:601-608 